\title{
Model ecosystem approach to estimate community level effects of radiation
}

\author{
M. Doi ${ }^{1}$, I. Kawaguchi ${ }^{1}$, N. Tanaka ${ }^{1}$, S. Fuma ${ }^{1}$, N. Ishii ${ }^{1}$, K. Miyamoto $^{1}$, \\ H. Takeda ${ }^{1}$ and Z. Kawabata ${ }^{2}$ \\ ${ }^{1}$ Center for Radiation Safety, National Institute of Radiological Sciences, 4-9-1, Anagawa, \\ Inage Chiba 263-8555, Japan \\ ${ }^{2}$ Center for Ecological Research, Kyoto University, Hirano-cho, Otsu, Shiga, Japan
}

\begin{abstract}
Mathematical computer model is developed to simulate the population dynamics and dynamic mass budgets of the microbial community realized as a self sustainable aquatic ecological system in the tube. Autotroph algae, heterotroph protozoa and saprotroph bacteria live symbiotically with interspecies' interactions as predator-prey relationship, competition for the common resource, autolysis of detritus and detritus-grazing food chain, etc. The simulation model is the individual-based parallel model, built in the demographic stochasticity, environmental stochasticity by dividing the aquatic environment into patches.

Validity of the model is checked by the multifaceted data of the microcosm experiments. In the analysis, intrinsic parameters of umbrella endpoint regarding to lethality are manipulated at the individual level, and tried to find the population level, community level especially focused on predator-prey relationship, and revealed the indirect effect of chronic exposure of radiation on the probability of Tetrahymena's extinction.
\end{abstract}

\section{INTRODUCTION}

Environment is a complex system with complicated webs of inter-species, intra-species, species-to-habitats interactions. Holistic and systematic views are essential to estimate the ecological impact assessments of stressors, including radiation and radioactive materials in the environment, but also, we should have a bird view to look down the complex system to identify "most essential and crucial structures and factors" which hold the key to the sustainability of the ecological system.

To link radiation research to population and ecosystem ecology is very fruitful, because eco toxicological assessments have widely introduced the ecological methodologies and models, and some of which are highly suggestive. For the environmental protection from the effects of radiation, we should draw a simplified picture and prospectus of the intra-species and inter-species interactions.

To understand the basic essential ecological functions, we should observe much simpler ecosystem. The artificial closed experimental ecosystem is studied in this study as a simplified sustainable reference ecosystem.

\section{MATERIAL AND METHODS}

\subsection{Reference microcosm}

Closed experimental aquatic Microbial ecosystem (microcosm), developed by Prof. Kawabata, Center for Ecological Research, Kyoto Univ. [1] is selected as a reference ecosystem in this study. The microcosm consists of heterotroph ciliate protozoa, Tetrahymena thermophila B as a consumer, autotroph 
flagellate algae, Euglena gracilis $\mathrm{Z}$ as a producer and saprotroph bacteria, Escherichia coli DH5 as a decomposer The culture medium is $10 \mathrm{ml}$ of water with inorganic salt and initial organic nutrients, and held in the airtight test tube. It is cultured statically with fluorescent lamps under $2500 \mathrm{~lx}$ and 12 hrs light-dark cycle at $25{ }^{\circ} \mathrm{C}$. The symbiosis among microbes is self-organized by realizing material cycle and sustained for more than 2 years after inoculation. The system can not afford to lose anyone of the microbes to maintain its sustainability. Material cycles in the microcosm and inhibitory effects of metabolites, especially $\mathrm{NH}_{3}$ in the environment were revealed [2].

\subsection{Ecotoxicological experiment}

Eco-toxicological tests with the microcosm were carried out by Fuma et al. since 1996. Showed impacts of Co-60 gamma ray and chronic exposure of inorganic heavy metal compounds on population dynamics of microbes in the test tube. Ecological evaluation of gadolinium and other heavy metals toxicity, acute exposure of Co-60 gamma radiation, and parallel effect of acidification and radiation exposure were estimated by using the aquatic microcosm [3-5].

\subsection{Individual-based simulation model for the Kawabata-microcosm}

To answer the query “if each individual is protected well, are they protected as population?”, we should link the individual level effects, both positive and negative to the population level ones. As a modelling of the microcosm, the individual-based bottom-up computer simulation program is developed by referring to theories in mathematical biology and ecology.

In the individual-based model, microbial constituents are defined as actors of crucial functions to the sustainability of the system. It is based on the experimental data on physiology, morphology and pathology for each constituents, and by the heuristic approach, we can analyse the simulation results to understand the systematic mechanism of the microcosm much better [6-7 ]. Illustration of the concept of the simulation is shown in Figure 1.

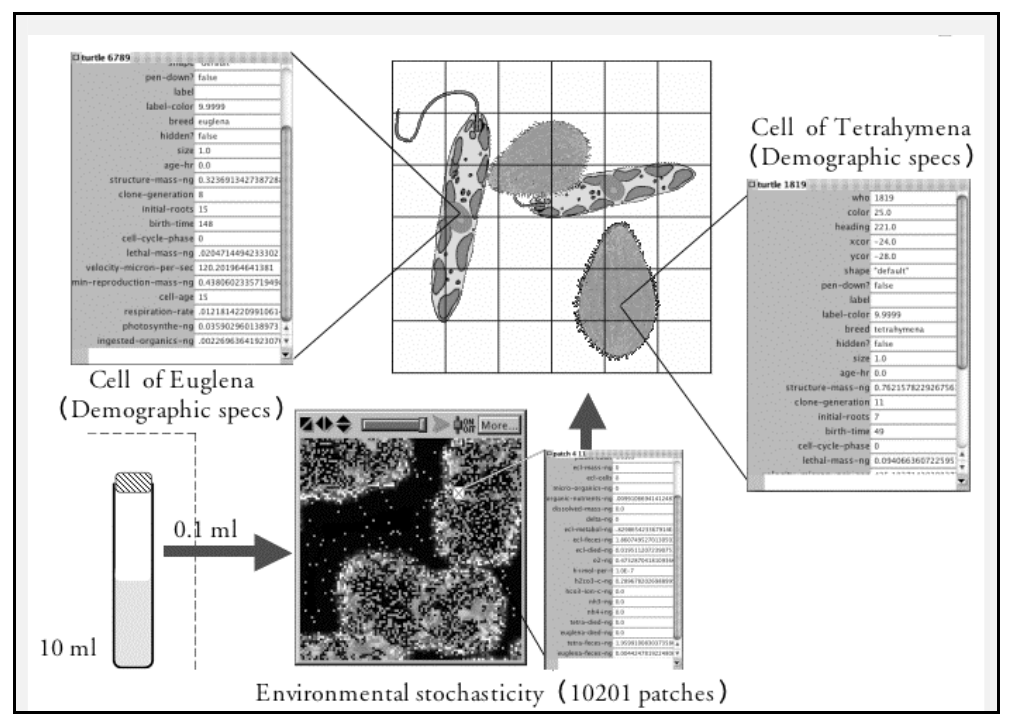

Figure 1. Illustration of the concept of individual-based modelling. 


\subsection{Impact of radiation on Predator-prey relationship in the microcosm}

One of the direct intra-species interaction in the microcosm is the grazing of Tetrahymena, which eats E. coli. Tetrahymena is a heterotroph protozoa, which may afford a grazing pressure on the E.coli, which may contribute the sustainability of E.coli by avoiding explosive growth and following extinction of E. coli. E.coli is a saprotroph bacteria, which may contribute the recycle of metabolites and dead bodies by fermentation.

As a grazing strategy, optimal foraging was adopted in the simulation by supposing the spatial distribution of E. coli as "negative binomial" [8]. Experimental results of two species subsystem culture and simulation results are shown in Figure. 2.

Predator-prey relationship may follow the well-known following equations, $x$ as a population of prey (E. coli) and $y$ as a population of predator (Tetrahymena).

$$
\begin{gathered}
\frac{d x}{d t}=r x\left(1-\frac{x}{K}\right)-\frac{a x}{1+h x} y-m x \\
\frac{d y}{d t}=\frac{x}{1+h x} b y-c y
\end{gathered}
$$

where $r$ is intrinsic growth rate of prey, $K$ is a carrying capacity of prey. $a$ and $h$ is a parameter of prey related to grazing efficiency of predator. $b$ is a parameter for assimilation of predator, and $c$ is a death rate of predator. Additional excess mortality given by radiation on the prey, E. coli is defined as $m$. Solve equations 1 and 2 at the equilibrium condition, equilibrium level of population of prey (E. coli), $x_{E q}$ and predator (Tetrahymena), $y_{E q}$ are given as follows.

$$
\begin{gathered}
x_{E q}=\frac{c}{b-h c}(>0) \quad b>h c \\
y_{E q}=r \frac{b}{a(b-h c)}\left(1-\frac{c}{K(b-h c)}\right)-m \frac{b}{a(b-h c)}
\end{gathered}
$$

As shown in Equation (3), equilibrium population of prey, E-coli does not influenced by excess lethality rate, " $m$ ", which is defined as a direct impact on E. coli by radiation. In stead, as shown in Equation (4), equilibrium population of predator, Tetrahymena decreases as " $m$ ” increases. This is a result of intra-species interaction. That is to say, radiation impact is on the individual of E. coli directly, but as a population, it is shifted to the less growth of Tetrahymena by starvation, and compensated by the reduction of grazing pressure due to the reduction of tetrahymena's population.

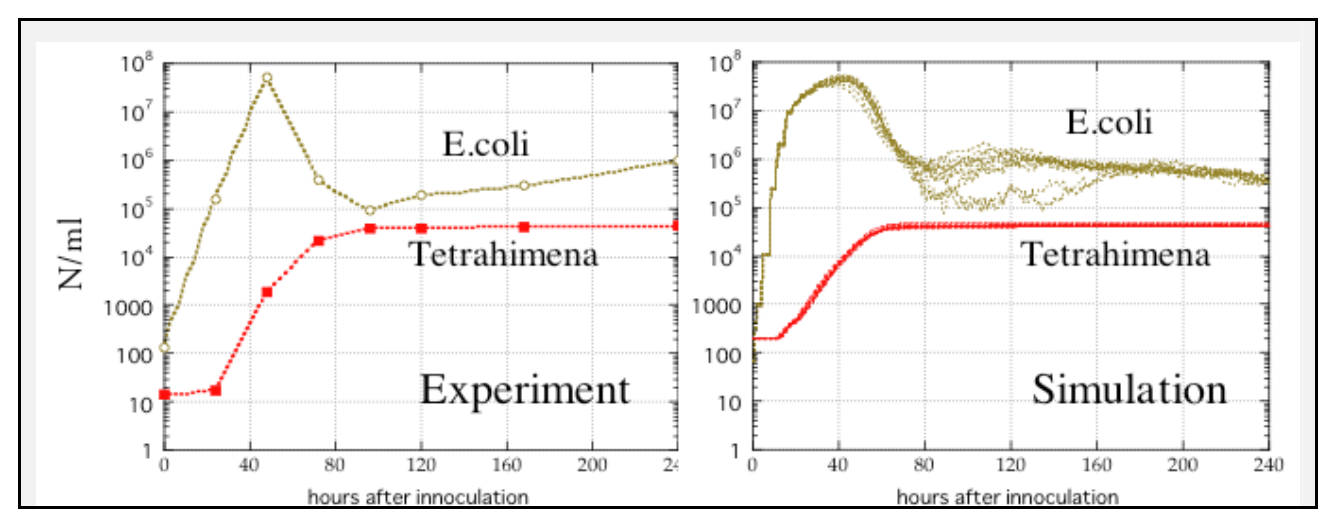

Figure 2. Experimental results of two species subsystem culture and simulation results. 


\subsection{Extrapolation from acute-high dose exposure to chronic-low dose exposure}

Because of data gap of dose-effect relatiopship for the chronic low dose and dose rate exposure, chronic dealth rate of $\mathrm{E}$. coli, of which $50 \%$ acute lethal dose is estimated to $20 \mathrm{~Gy}$, was estimated by extrapolation from acute high dose-effect relationship as shown in Figure. 3. Advisability of extrapolation from acute high dose effect to chronic low dose effect shall be discussed independently. Since 50\% acute letheal dose of Tetrahymena is estimated to $2000 \mathrm{~Gy}$, its low dose rate effects were neglected in this study.

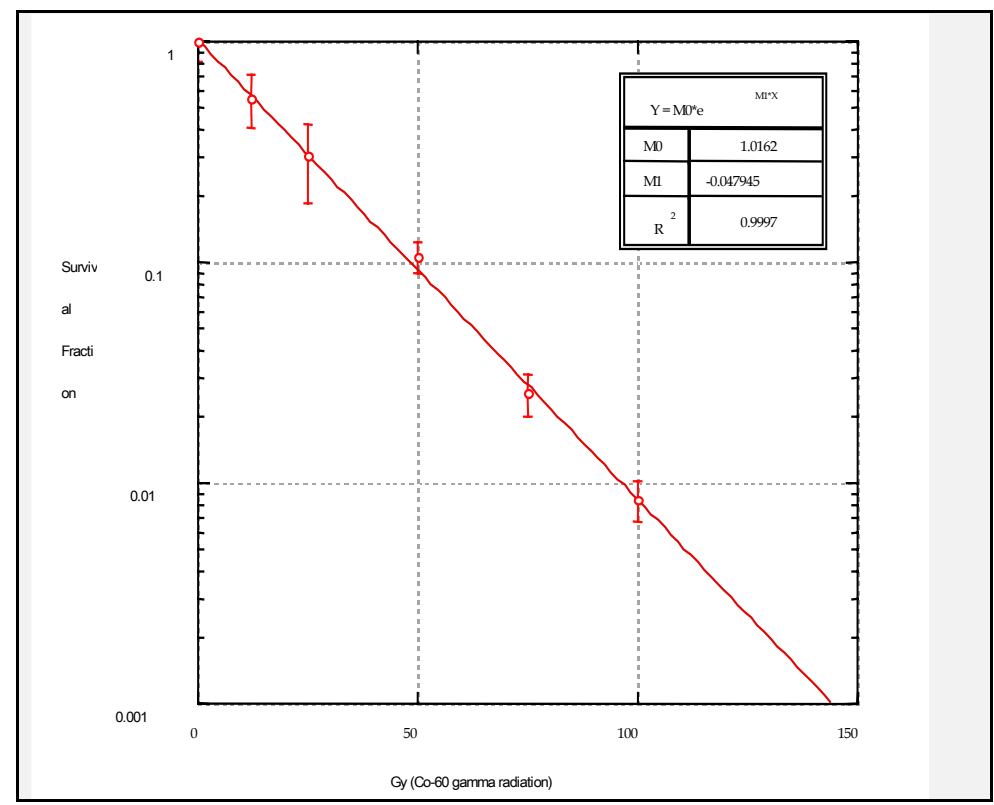

Figure 3. Suevival curve of E. coli by acute Co-60 gamma radiation exposure.

\section{RESULTS AND DISCUSSION}

Simulation results of subsystem culture of Tetrahymena and E. coli was shown in Figure 4. Population dynamics of Tetrahymena as predator and E.coli as prey simulated relatively well. Both microbes discharge $\mathrm{NH}_{3}$ as a metabolite, which may have inhibitory effects on themselves [2]. Because of quantitative data gap, these negative effects are not included in the simulation. It is expected that inhibitory effects on E.coli may shift to Tetrahymena through predator- prey relationship (point "A" should shift downward), and direct inhibitory effects on Tetrahymena may act positive to E.coli (point "B" should shift upward). This is left for future assignment. As a reference, experimental results of microcosm and subsystem cultures were shown in Figure 5. 


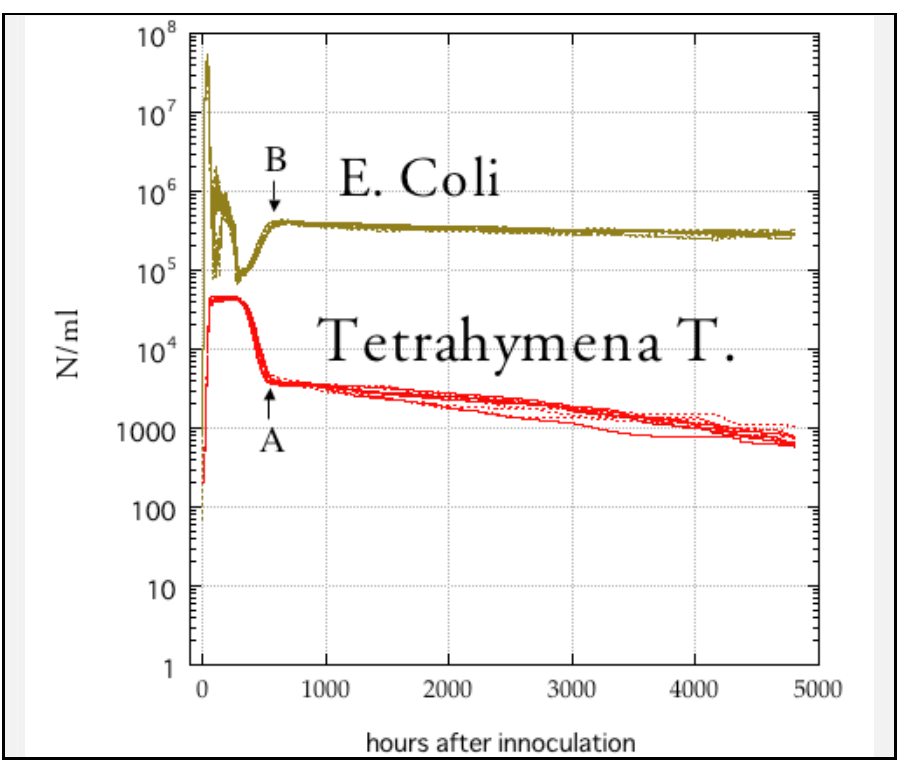

Figure 4. Population dynamics of heterotroph protozoa (predator, Tetrahymena, T.) and saprotroph bacteria (prey, E. coli) simulated by SIM-COSM. Both microbes discharge $\mathrm{NH}_{3}$ as a metabolite, which may have inhibitory effects on themselves [2]. Because of quantitative data gap, these negative effects are not included in the simulation. Inhibitory effects on E.coli may shift to Tetrahymena through predator- prey relationship (point "A" should shift downward), and direct inhibitory effects on Tetrahymena may act positive to E.coli (point "B" should shift upward).

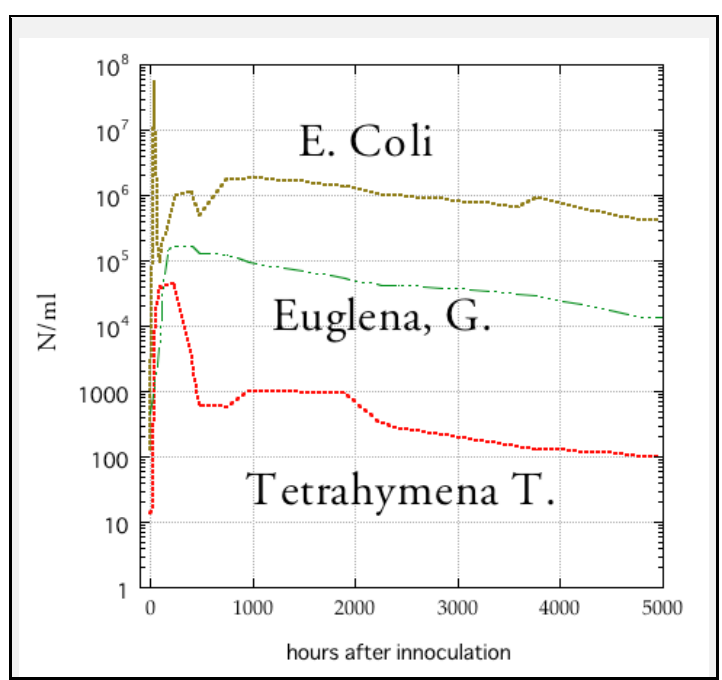

a) Producer - consumer - decomposer system

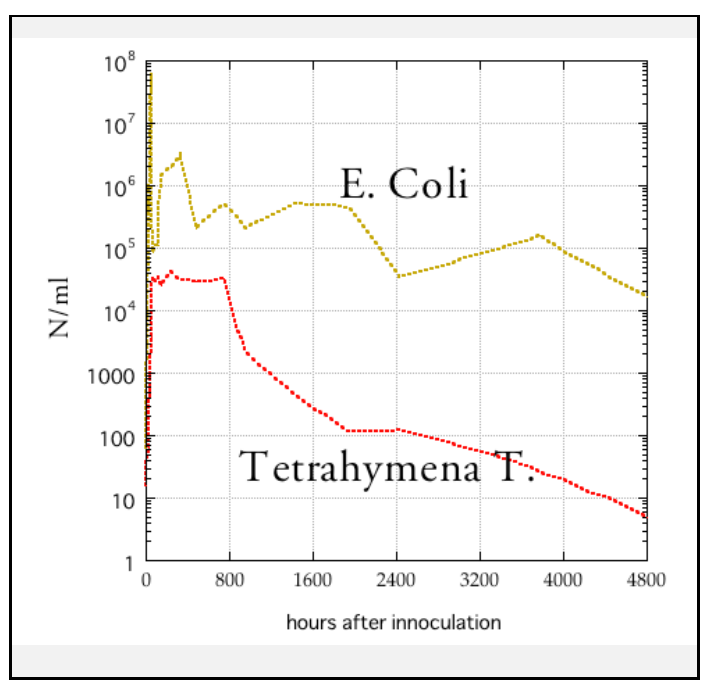

b) Consumer - decomposer system

Figure 5. Population dynamics of microbes observed in the microcosm and its subsystem by the experiment [3]. 


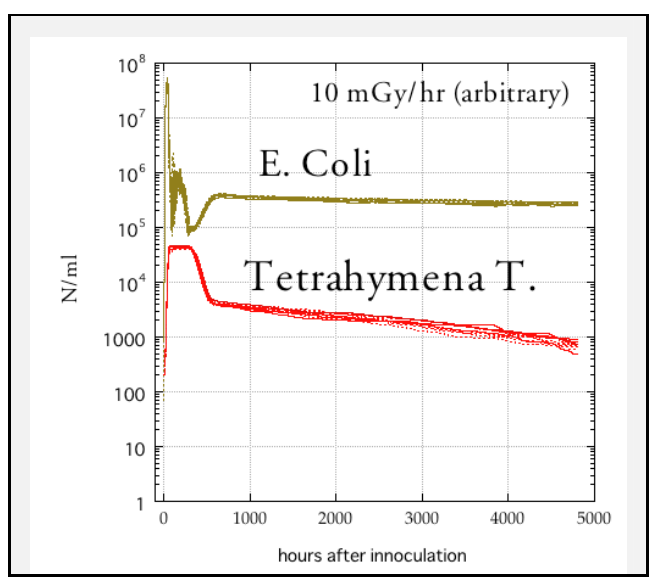

a) Added excess death rate: $0.035 \% / \mathrm{hr}$

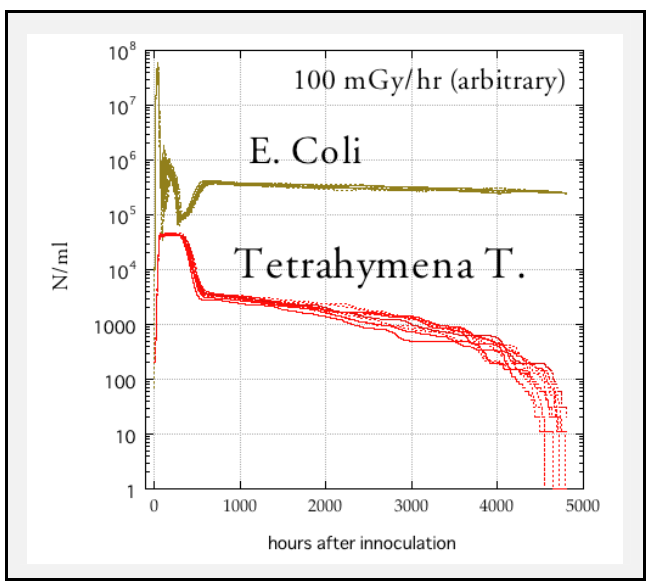

c) Added excess death rate: $0.35 \% / \mathrm{hr}$

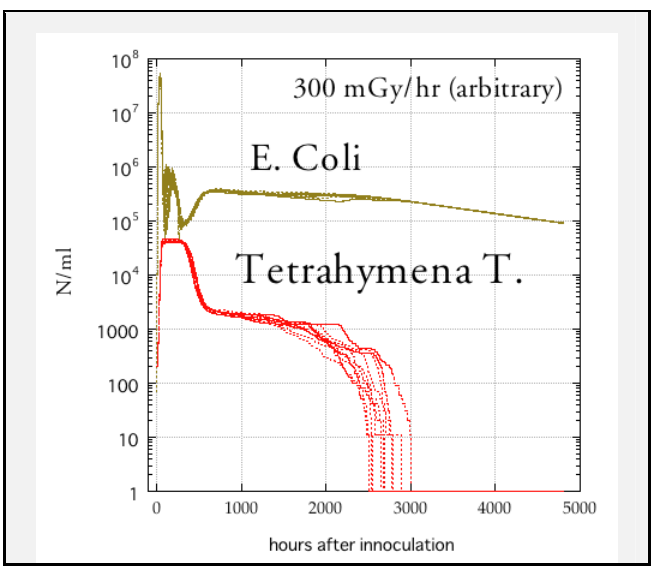

e) Added excess death rate: $1.0 \% / \mathrm{hr}$

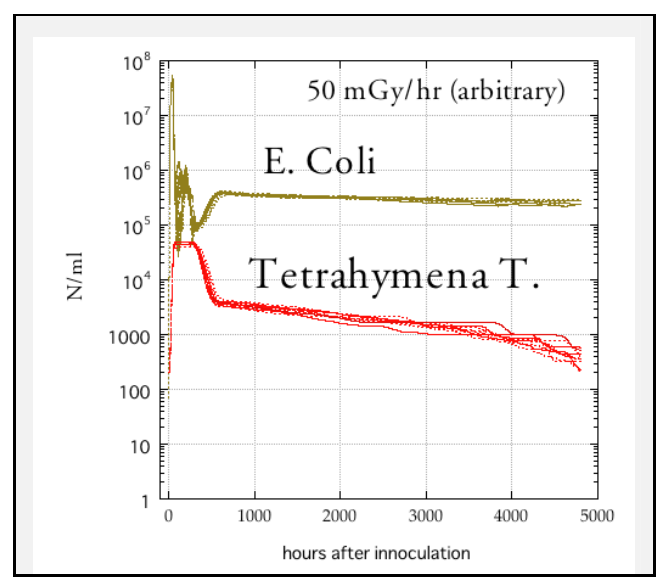

b) Added excess death rate: $0.17 \% / \mathrm{hr}$

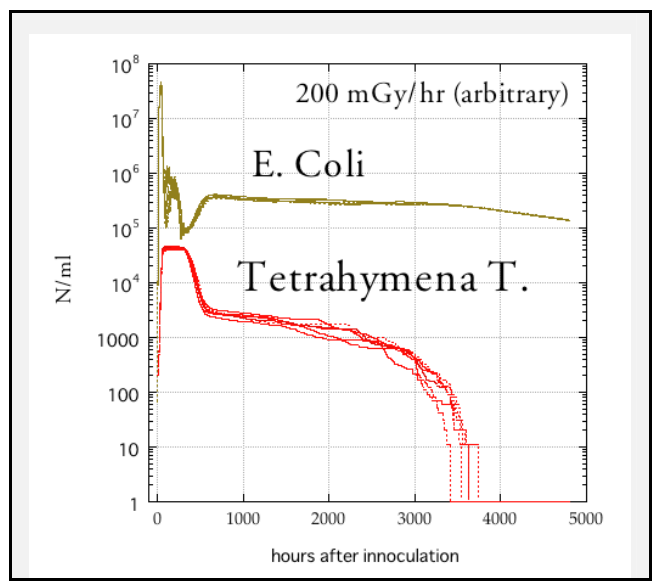

d) Added excess death rate: $0.69 \% / \mathrm{hr}$

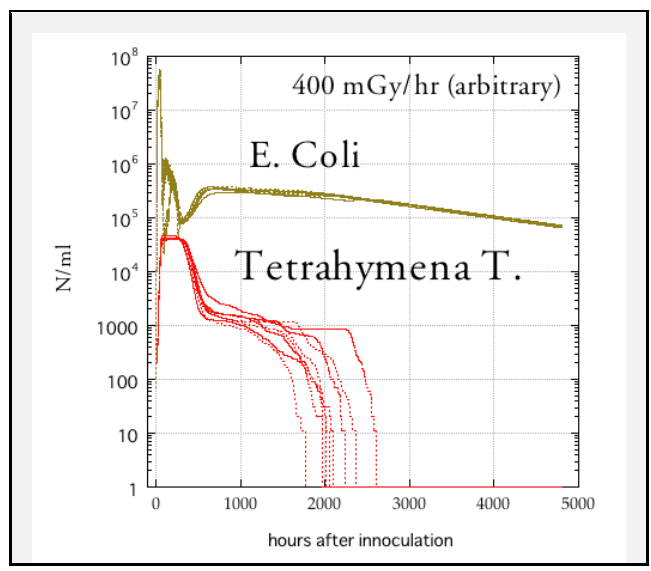

f) Added excess death rate: $1.4 \% / \mathrm{hr}$

Figure 6. Impact of chronic gamma radiation exposures on the population dynamics of heterotroph protozoa (predator, Tetrahymena, T.) and saprotroph bacteria (prey, E. coli) simulated by SIM-COSM. Each dose rate in the figure are arbitrarily estimated to each death rate of E.coli (m-value), on the basis of extrapolation from dose-effect relationship of E.coli at acute high dose exposures shown in Figure 3. 
Population dynamics of 6 different death date (m-value) of E. coli (expected dose rate of $10-400 \mathrm{mGy} / \mathrm{hr}$ ) predicted by the simulation were summarised in Figure 6. Even though, direct effect of radiation was given to E. coli at the individual level, their impacts were shifted to their predator's population level effect, i.e., reduction of carrying capacity and extinction as the results of less growth and starvation at the individual level. Simple predator-prey relationship in the simulation suggested the indirect impacts on the population of "radio-resistant predator". It also found that mall increase of death rate which induced by radiation is a direct effect on the individual of "radio-sensitive prey", but not on its population. These findings may suggest that species that depend their intrinsic growth on the "radiosensitive" species could be a candidate of index organism to be concerned to show the impact of radiation and radioactivity in the environment.

\section{CONCLUSIVE REMARKS}

Mathematical computer model is developed to simulate the population dynamics and dynamic mass budgets of the microbial community realized as a self sustainable aquatic ecological system in the tube. In the analysis, intrinsic parameters of umbrella endpoint regarding to lethality are manipulated at the individual level, and tried to find the population level, community level especially focused on predator-prey relationship, and revealed the indirect effect of chronic exposure of radiation on the probability of Tetrahymena's extinction.

\section{Acknowledgments}

The authors are grateful to Professor Yoh Iwasa (Kyusyu University, Japan) and Dr. Shuichi Shikano (Tohoku University, Japan) for useful discussions and encouragement.

\section{References}

[1] Z. Kawabata, K, Matsui, K, Okazaki, M. Nasu, N. Nakano, T. Sugai, J. Protozool., Res. 5 (1995), pp23-26.

[2] S. Shikano, Z. Kawabata, Hydrobiologia, 436 (2000) pp209-216.

[3] S. Fuma, H. Takeda, K. Miyamoto, K. Yanagisawa, Y. Inoue, N. Ishii, K. Sugai, C. Ishii, Z. Kawabata, Bulletin. Environ. Cont. and Toxicol. 66 (2001) pp.231-238.

[4] S. Fuma, N. Ishii, H. Takeda, K. Miyamoto, K. Yanagisawa, Y. Ichimasa, M. Saito, Z. Kawabata, G. G. Polikarpov, J. Env. Rad. 67 (2003) pp 1-14.

[5] S. Fuma, N. Ishii, H. Takeda, Z. Kawabata, Y.Ichimasa, Radioisotopes 51 (2002) pp 204-213.

[6] M. Doi ; OECD/NEA report, ISBN 92-64-09969-7 (2002) pp131-143.

[7] M. Doi, N. Tanaka, Z. Kawabata, IAEA-CN-109 (2003) pp 70-73.

[8] Y. Iwasa, M. Higashi, N. Yamamura, The Ame. Natul. 117 (1981) pp710-723. 